\title{
A clinical comparison of continuous interscalene brachial plexus block with different basal infusion rates of $0.2 \%$ ropivacaine for shoulder surgery
}

\author{
Chun Woo Yang ${ }^{1}$, Sung Mee Jung ${ }^{1}$, Hee Uk Kwon ${ }^{1}$, Choon-Kyu Cho ${ }^{1}$, Jin Woong Yi ${ }^{2}$, Chul Woung Kim³ , \\ Jong-Kwon Jung ${ }^{4}$, and Young Mi $\mathrm{An}^{4}$ \\ Departments of ${ }^{1}$ Anesthesiology and Pain Medicine, ${ }^{2}$ Orthopaedic Surgery, School of Medicine, Konyang University, Daejeon, \\ ${ }^{3}$ Department of Preventive Medicine, School of Medicne, Chungnam National University, Daejeon, ${ }^{4}$ Department of Anesthesiology \\ and Pain Medicine, Inha University College of Medicine, Incheon, Korea
}

Background: A continuous interscalene brachial plexus block is a highly effective postoperative analgesic modality after shoulder surgery. However, there is no consensus regarding the optimal basal infusion rate of ropivacaine for a continuous interscalene brachial plexus block. A prospective, double blind study was performed to compare two different basal rates of $0.2 \%$ ropivacaine for a continuous interscalene brachial plexus block after shoulder surgery.

Methods: Sixty-two patients receiving shoulder surgery under an interscalene brachial plexus block were included. The continuous interscalene brachial plexus block was performed using a modified lateral technique with $30 \mathrm{ml}$ of 0.5\% ropivacaine. Surgery was carried out under an interscalene brachial plexus block or general anesthesia. After surgery, the patients were divided randomly into two groups containing 32 each. During the first $48 \mathrm{~h}$ after surgery, groups R8 and R6 received a continuous infusion of $0.2 \%$ ropivacaine at $8 \mathrm{ml} / \mathrm{h}$ and $6 \mathrm{ml} / \mathrm{h}$, respectively. The pain scores at rest and on movement, supplemental analgesia, motor block, adverse events and patient's satisfaction were recorded.

Results: The pain scores, supplemental analgesia, motor block, adverse events and patient's satisfaction were similar in the two groups.

Conclusions: When providing continuous interscalene brachial plexus block after shoulder surgery, $0.2 \%$ ropivacaine at a basal rate of $8 \mathrm{ml} / \mathrm{h}$ or $6 \mathrm{ml} / \mathrm{h}$ produces similar clinical efficacy. Therefore, decreasing the basal rate of CISB is more appropriate considering the toxicity of local anesthetics. (Korean J Anesthesiol 2010; 59: 27-33)

Key Words: Continuous interscalene block, Ropivacaine, Shoulder surgery.

Received: March 22, 2010. Revised: 1st, April 10, 2010; 2nd, April 19, 2010. Accepted: April 27, 2010.

Corresponding author: Sung Mee Jung, M.D., Department of Anesthesiology and Pain Medicine, School of Medicine, Konyang University, 685, Gasuwon-dong, Seo-gu, Daejeon 302-718, Korea. Tel: 82-42-600-9316, Fax: 82-42-545-2132, E-mail: applejsm@hotmail.com

(c) This is an open-access article distributed under the terms of the Creative Commons Attribution Non-Commercial License (http:// creativecommons.org/licenses/by-nc/3.0/), which permits unrestricted non-commercial use, distribution, and reproduction in any medium, provided the original work is properly cited. 


\section{Introduction}

Surgery in the shoulder region is often associated with severe postoperative pain that may require opioids for several days [1]. A single-shot interscalene brachial plexus block (ISB) can provide pain relief for up to $18 \mathrm{~h}$, after which the patient is reliant on conventional analgesia with its associated side-effects. Compared to IV patient-controlled analgesia (PCA) for open shoulder surgery, prospective, randomized, controlled trials have demonstrated that the use of a continuous interscalene brachial plexus block (CISB) reduces the postoperative requirements for opioids and provides better analgesia, reduced opioid-related side effects, and better patient satisfaction for at least the first $48 \mathrm{~h}$ after surgery $[1,2]$.

Ropivacaine is a long-acting amide local anesthetic with a similar structure and clinical profile to bupivacaine but with less associated toxicity at comparable doses [3]. For this reason, ropivacaine is the preferred local anesthetic for peripheral nerve blocks and continuous peripheral nerve infusions in many institutions. A comparison of CISB with $0.2 \%$ ropivacaine versus $0.15 \%$ bupivacaine revealed equivalent analgesia in both groups but significantly less motor block with ropivacaine [4].

However, there is no consensus regarding the optimal basal infusion rate of ropivacaine for CISB. As a result, many different basal infusion rates for CISB have been used. Ilfeld et al. [5] reported that providing patients with a ropivacaine $(0.2 \%)$ CISB at $8 \mathrm{ml} / \mathrm{h}$ produced potent analgesia after moderate to severely painful shoulder surgery, whereas lower infusion rates were often inadequate.

At our institution, a regimen of $0.2 \%(2 \mathrm{mg} / \mathrm{ml})$ ropivacaine at $6 \mathrm{ml} / \mathrm{h}$, supplemented with on-demand $3 \mathrm{ml} / 20 \mathrm{~min}$ boluses are commonly used for CISB. Low background infusions are advantageous for CISB, in that they carry a potential risk of local anesthetic toxicity and enable longer potent analgesia provided by limited volume pumps.

This prospective, double blind study examined the quality of postoperative analgesia, supplemental analgesia, motor block, adverse events and patient's satisfaction of CISB with $0.2 \%$ ropivacaine at a basal rate of either $8 \mathrm{ml} / \mathrm{h}$ or $6 \mathrm{ml} / \mathrm{h}$ for shoulder surgery.

\section{Materials and Methods}

With ethics committee approval and written informed consent, 64 ASA physical status I-III in-patients undergoing shoulder surgery with an interscalene brachial plexus block or general anesthesia were examined. The exclusion criteria were patients receiving chronic analgesic therapy, as well as patients with severe bronchopulmonary disease, neuropathy or an allergy to amide local anesthetics, nonsteroidal anti-inflammatory drugs or opioids.

Before the block procedure, standard monitors were placed and the patients received $0.1 \mu \mathrm{g} / \mathrm{kg}$ of sufentanil intravenously. All nerve blocks were performed by, or under the supervision of, an experienced anesthesiologist. The ISB was performed in all patients through a catheter using modified lateral techniques before sedation or the induction of general anesthesia. Anatomical surface landmarks of the neck were identified and marked with a surgical marking pen. Formal sterile techniques were used. The interscalene brachial plexus was identified using a nerve stimulator (Stimuplex ${ }^{\circledR}$-DIG, B/Braun, Germany) connected to the proximal end of the metal inner needle of a plastic cannula (Contiplex ${ }^{\circledR}$ A, B/Braun, Germany).

The stimulation frequency was set to $1 \mathrm{~Hz}$ and a pulse duration of $0.1 \mathrm{~ms}$, while the intensity of the stimulating current, which was initially set to $1 \mathrm{~mA}$, was decreased progressively to $\leq 0.6 \mathrm{~mA}$ after the appropriate motor response had been observed. A 22-gauge catheter was introduced $4-5 \mathrm{~cm}$ into the plexus sheath through a cannula. All patients received local anesthetics through the catheter. $0.5 \%$ ropivacaine $30 \mathrm{ml}$ were injected slowly in $5 \mathrm{ml}$ aliquots with multiple negative aspirations of blood. The catheter was tunneled subcutaneously over $3-4 \mathrm{~cm}$ through an 18-gauge IV needle and fixed to the skin with a tight suture.

Surgical anesthesia was defined as the complete loss of cold sensation at the skin dermatomes involved in the surgical field (from C5-6) and an inability to abduct the arm and flex the forearm against gravity at the shoulder and elbow joints, respectively. The block was considered to have failed if the block was not complete $45 \mathrm{~min}$ after the injection.

The general anesthetic technique used (when necessary) was standard for all patients. After the block was complete, induction was performed with $3 \mu \mathrm{g} / \mathrm{ml}$ propofol (Fresofol ${ }^{\circledR} 2 \%$, Fresenius Kabi, Germany) using a target-controlled infusion (TCI) system (Orchestra Base Primea ${ }^{\circledR}$, Fresenius Vial, France). At the same time, sufentanil was started using the same TCI system used for propofol. Propofol at an effect site concentration of $3 \mu \mathrm{g} / \mathrm{ml}$ and $0.1 \mathrm{ng} / \mathrm{ml}$ sufentanil were administered. Tracheal intubation was facilitated using $0.6 \mathrm{mg} / \mathrm{kg}$ rocuronium administered within 3 min before tracheal intubation. Anesthesia was maintained with propofol $2-3 \mu \mathrm{g} / \mathrm{ml}$, sufentanil $0.1 \mathrm{ng} / \mathrm{ml}$. Those not under general anesthesia received sufentanil $0.2-0.3 \mu \mathrm{g} / \mathrm{kg}$ IV and a propofol infusion at $50 \mu \mathrm{g} / \mathrm{kg} / \mathrm{min}$ for sedation. Supplemental oxygen was administered throughout the procedure at $6 \mathrm{~L} / \mathrm{min}$ through a mask.

In the postanesthetic care unit (PACU), the patients were divided randomly into two groups containing 32 each. During the first $48 \mathrm{~h}$ postoperatively, Group R8 received a continuous infusion of $0.2 \%$ ropivacaine at $8 \mathrm{ml} / \mathrm{h}$ plus boluses of $3 \mathrm{ml}$ with a lockout time of $20 \mathrm{~min}$ through the interscalene catheter using 
a portable battery-powered pump (Accumate ${ }^{\circledR} 1000$, Wooyoung Medical, Korea). Group R6 received a continuous infusion of the same solution at $6 \mathrm{ml} / \mathrm{h}$ plus boluses of $3 \mathrm{ml}$ with a lockout time of $20 \mathrm{~min}$. In addition, rescue analgesia with $60 \mathrm{mg}$ of IM diclofenac was available on demand. The demographic parameters, type and duration of the surgical procedure were similar in the two groups (Table 1 and 2).

Pain at rest and on movement, as well as the motor function of the operated arm was assessed at the PACU and at 24 and $48 \mathrm{~h}$ after surgery. The pain intensity was assessed using a 10$\mathrm{cm}$ visual analog scale $(0 \mathrm{~cm}=$ no pain; $10 \mathrm{~cm}=$ worst pain imaginable) for pain at rest and during passive movement $\left(90^{\circ}\right.$ abduction) of the shoulder. The motor function of the operated arm was evaluated by asking the patient to squeeze the hands of a blinded observer with both hands, who scored the motor function using a three-point scale as follows: $0=$ no motor block, similar strength in both hands; $1=$ partial motor block, operated hand weaker than the non-operated one; $2=$ complete motor block, unable to squeeze with the operated hand. Supplemental analgesia, ropivacaine consumption, sleep disturbance, adverse events, and satisfaction score ( $1=$ satisfied; if needed will undergo the same procedure again in the future; 2 = unsatisfied; if needed in the future, will ask for a

Table 1. Demographic Data

\begin{tabular}{lcc}
\hline & Group R8 (n=31) & Group R6 (n=31) \\
\hline Age (years) & $54 \pm 16$ & $55 \pm 15$ \\
Male/female & $20 / 11$ & $18 / 13$ \\
Height (cm) & $163 \pm 9$ & $162 \pm 10$ \\
Weight (kg) & $64 \pm 10$ & $63 \pm 10$ \\
ASA physical status (I/II/III) & $12 / 18 / 1$ & $11 / 19 / 1$ \\
Duration of surgery (min) & $140 \pm 44$ & $138 \pm 59$ \\
\hline
\end{tabular}

Values are the mean \pm SD. Group R8: continuous interscalene brachial plexus block with $0.2 \%$ ropivacaine at a rate of $8 \mathrm{ml} / \mathrm{h}$, Group R6: continuous interscalene brachial plexus block with $0.2 \%$ ropivacaine at a rate of $6 \mathrm{ml} / \mathrm{h}$.

Table 2. Surgical Procedures

\begin{tabular}{lcc}
\hline & Group R8 $(\mathrm{n}=31)$ & Group R6 $(\mathrm{n}=31)$ \\
\hline A/S RCR & $10(32)$ & $11(35)$ \\
A/S capsuloplasty & $5(16)$ & $4(13)$ \\
A/S debridement & $2(6)$ & $4(13)$ \\
Open RCR & $2(6)$ & $1(3)$ \\
SA & $4(13)$ & $1(3)$ \\
ACJ reconstruction & $2(6)$ & $2(6)$ \\
Osteosynthesis & $6(19)$ & $8(26)$ \\
\hline
\end{tabular}

Values are the number of patients (\%). Group R8: continuous interscalene brachial plexus block with $0.2 \%$ ropivacaine at a rate of 8 $\mathrm{ml} / \mathrm{h}$, Group R6: continuous interscalene brachial plexus block with $0.2 \%$ ropivacaine at a rate of $6 \mathrm{ml} / \mathrm{h}$. A/S: arthroscopic, RCR: rotator cuff repair, SA: shoulder arthroplasty, ACJ: acromioclavicular joint. different analgesia technique.) were recorded at the end of the study period. All data was collected by an anesthesiologist who was neither involved in the administration of anesthesia nor in the patient care in the recovery room.

Using type I $(\alpha)$ and type II ( $\beta$ ) errors of 0.05 and 0.2 respectively, and considering a $20 \%$ difference in the average pain scores over a 48 hour period between the groups to be the minimum relevant difference, it was calculated that a sample of 25 patients per group would be necessary. Seven more patients per group were added to increase the power.

Statistical analysis was performed using Microsoft Excel Version 2007 (Microsoft Office Excel ${ }^{\circledR}$, Microsoft, USA). The normal distribution of the data was first evaluated using a Kolmogorov-Smirnov test. The normally distributed variables were analyzed using parametric methods. The data is reported as the mean $\pm \mathrm{SD}$, and the groups were compared using a Student's $t$-test. Otherwise, the variables were analyzed using nonparametric methods. The groups were summarized using the median $\left(10^{\text {th }}-90^{\text {th }}\right.$ percentiles $)$ and the groups were compared using a Mann-Whitney rank-sum test. A Fisher's exact test was used to compare the categorical data where appropriate. A P value $<0.05$ was considered significant.

\section{Results}

62 patients enrolled completed the study. One patient in group R6 was excluded because no motor response could be elicited. One patient in group R8 was excluded due to chest discomfort caused by phrenic nerve paresis. In one patient in group R8 and two patients in group R6, the interscalene catheter was accidentally pulled out $24 \mathrm{hrs}$ after surgery. Statistical analyses were performed without these patients at the missing time points. Two patients in group R8 and one in group R6 underwent surgery with general anesthesia. Table 3 lists the elicited motor responses.

Fig. 1 and 2 show the VAS scores at rest and on shoulder abduction. The pain scores in the PACU at 24 and $48 \mathrm{~h}$ after surgery were similar in the two groups. In addition, the proportion of patients requiring rescue diclofenac analgesia

Table 3. Motor Response to Nerve Stimulation

\begin{tabular}{lcc}
\hline & Group R8 $(\mathrm{n}=30)$ & Group R6 (n=31) \\
\hline Deltoid muscle & $13(43)$ & $19(61)$ \\
Triceps muscle & $5(17)$ & $5(16)$ \\
Biceps muscle & $6(20)$ & $4(13)$ \\
Pectoralis major muscle & $6(20)$ & $3(10)$ \\
\hline
\end{tabular}

Values are the number of patients (\%). Group R8: continuous interscalene brachial plexus block with $0.2 \%$ ropivacaine at a rate of $8 \mathrm{ml} / \mathrm{h}$, Group R6: continuous interscalene brachial plexus block with $0.2 \%$ ropivacaine at a rate of $6 \mathrm{ml} / \mathrm{h}$. 


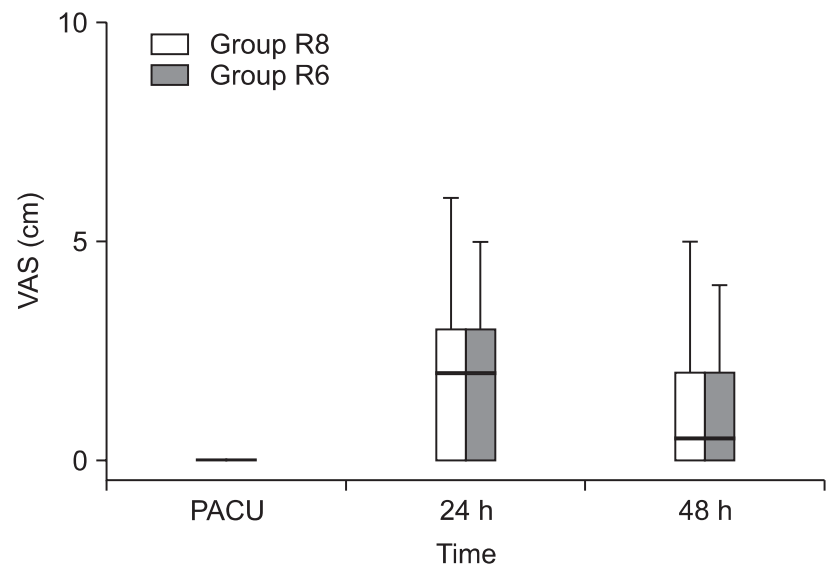

Fig. 1. Median and range for the visual analog scale pain scores at rest $(0 \mathrm{~mm}=$ no pain to $10 \mathrm{~mm}=$ worst pain imaginable $)$. Group R8: continuous interscalene brachial plexus block with $0.2 \%$ ropivacaine at a rate of $8 \mathrm{ml} / \mathrm{h}$, Group R6: continuous interscalene brachial plexus block with $0.2 \%$ ropivacaine at a rate of $6 \mathrm{ml} / \mathrm{h}$. PACU: postanesthetic care unit.

Table 4. Analgesic Effectiveness

\begin{tabular}{lcc}
\hline & Group R8 (n=31) & Group R6 (n=31) \\
\hline $\begin{array}{l}\text { Rescue analgesia } \\
\text { (with IM diclofenac) }\end{array}$ & & \\
POD & $14(45)$ & $14(45)$ \\
POD 1 & $11(35)$ & $10(32)$ \\
Sleep disturbance & & \\
POD & $13(42)$ & $10(32)$ \\
POD 1 & $6(19)$ & $6(19)$ \\
\hline
\end{tabular}

Values are the number of patients (\%). Group R8: continuous interscalene brachial plexus block with $0.2 \%$ ropivacaine at a rate of $8 \mathrm{ml} / \mathrm{h}$, Group R6: continuous interscalene brachial plexus block with $0.2 \%$ ropivacaine at a rate of $6 \mathrm{ml} / \mathrm{h}$. POD: postoperative day.

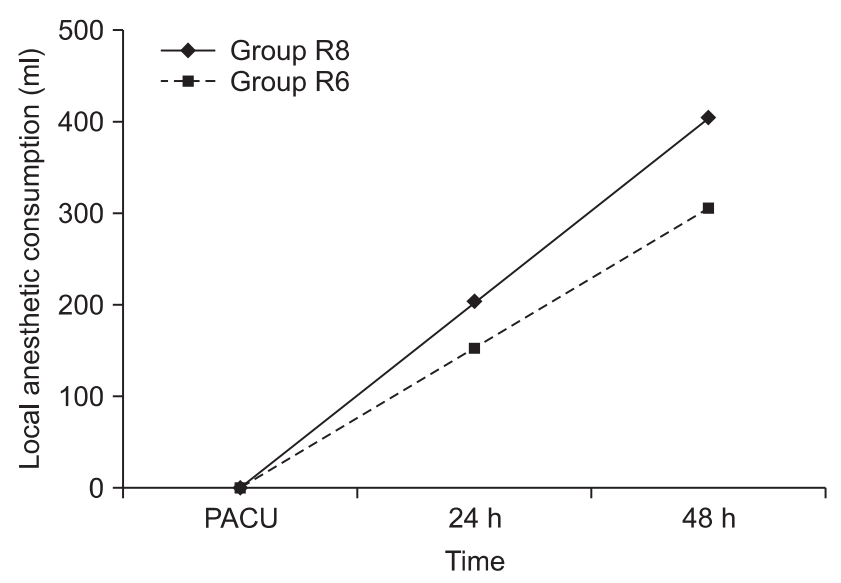

Fig. 3. Local anesthetic consumption. Group R8: continuous interscalene brachial plexus block with $0.2 \%$ ropivacaine at a rate of $8 \mathrm{ml} / \mathrm{h}$, Group R6: continuous interscalene brachial plexus block with $0.2 \%$ ropivacaine at a rate of $6 \mathrm{ml} / \mathrm{h}$. PACU: postanesthetic care unit.

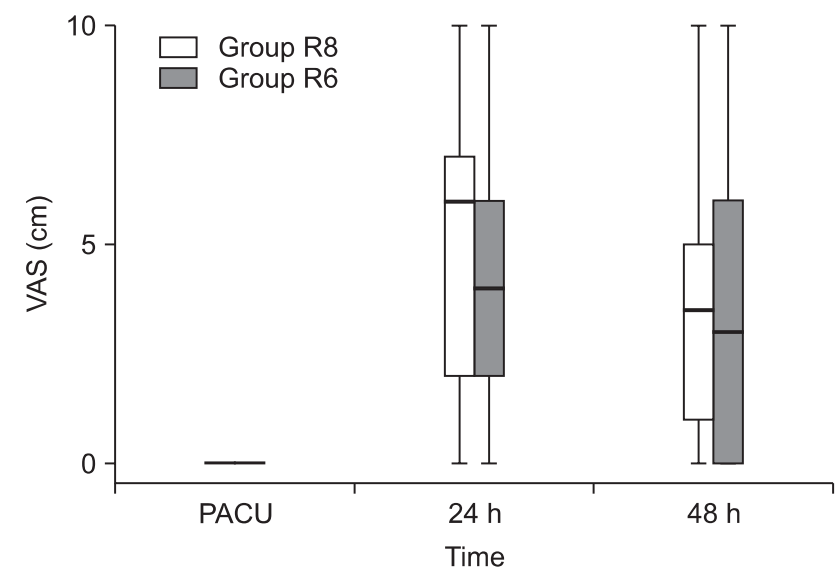

Fig. 2. Median and range for the visual analog scale pain scores during passive movement of the shoulder $(0 \mathrm{~cm}=$ no pain to $10 \mathrm{~cm}=$ worst pain imaginable). Group R8: continuous interscalene brachial plexus block with $0.2 \%$ ropivacaine at a rate of $8 \mathrm{ml} / \mathrm{h}$, Group R6: continuous interscalene brachial plexus block with $0.2 \%$ ropivacaine at a rate of $6 \mathrm{ml} / \mathrm{h}$. PACU: postanesthetic care unit.

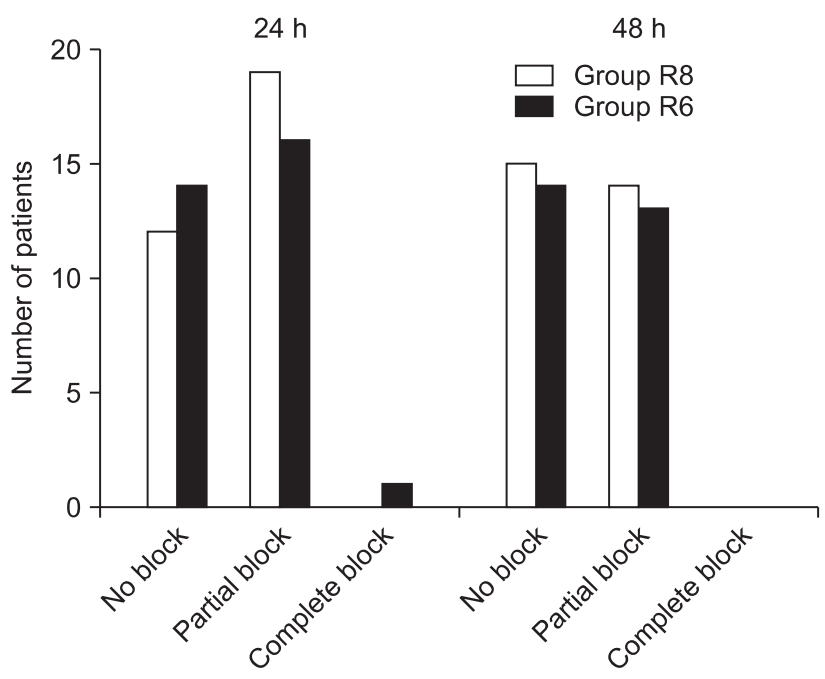

Fig. 4. Progression of the motor block in the operated limb during 48 $\mathrm{h}$ of CISB. Group R8: continuous interscalene brachial plexus block with $0.2 \%$ ropivacaine at a rate of $8 \mathrm{ml} / \mathrm{h}$, Group R6: continuous interscalene brachial plexus block with $0.2 \%$ ropivacaine at a rate of $6 \mathrm{ml} / \mathrm{h}$.

was also similar in the two groups (Table 4). There were no significant differences in sleep disturbances on postoperative days 0 and 1 (Table 4). The volume of the local anesthetic solution administered at $24 \mathrm{~h}$ and $48 \mathrm{~h}$ postoperatively was 204 \pm 18 and $404 \pm 31 \mathrm{ml}$ in group R8 and $152 \pm 11$ and $307 \pm 19 \mathrm{ml}$ in group R6, respectively (Fig. 3).

Fig. 4 shows the progression of the motor block in the operated limb during the study period. There were no significant differences in motor block between the two groups. 
Table 5. Adverse Events

\begin{tabular}{lcc}
\hline & Group R8 $(\mathrm{n}=31)$ & Group R6 (n=31) \\
\hline Nausea & $1(0.03)$ & $3(0.03)$ \\
Dyspnea & $4(0.13)$ & $2(0.06)$ \\
Hoarseness & $5(0.16)$ & $2(0.06)$ \\
Horner's syndrome & $14(0.45)$ & $14(0.45)$ \\
\hline
\end{tabular}

Values are the number of patients (\%). Group R8: continuous interscalene brachial plexus block with $0.2 \%$ ropivacaine at a rate of $8 \mathrm{ml} / \mathrm{h}$, Group R6: continuous interscalene brachial plexus block with $0.2 \%$ ropivacaine at a rate of $6 \mathrm{ml} / \mathrm{h}$.

Table 6. Patient's Satisfaction

\begin{tabular}{lcc}
\hline & Group R8 $(\mathrm{n}=28)$ & Group R6 $(\mathrm{n}=30)$ \\
\hline Unsatisfied & $3(11)$ & $2(7)$ \\
Satisfied & $25(89)$ & $28(93)$ \\
\hline
\end{tabular}

Values are the number of patients (\%). Group R8: continuous interscalene brachial plexus block with $0.2 \%$ ropivacaine at a rate of $8 \mathrm{ml} / \mathrm{h}$, Group R6: continuous interscalene brachial plexus block with $0.2 \%$ ropivacaine at a rate of $6 \mathrm{ml} / \mathrm{h}$.

Table 5 summarizes the adverse events during the study. No statistical difference was observed between the two groups. These adverse events were observed mainly during the immediate postoperative period.

The patients were not examined specifically for any long-term neurologic complications. However, neurological complications were reported by the surgeons in two patients in Group R8. One patient reported paresthesia in the first two fingers for 3 weeks, which resolved progressively. The other patient reported paresthesia in the areas of the musculocu-taneous and radial nerve for 4 weeks, which also resolved progressively.

There were no significant differences in patient's satisfaction between the groups (Table 6).

\section{Discussion}

This study demonstrated that after shoulder surgery, different basal infusion rates of CISB provide similar postoperative analgesia, supplemental analgesic requirements, motor block, side effects and high patient satisfaction during the infusion period.

Shoulder surgery is associated with severe postoperative pain. The options available to optimize postoperative pain control after shoulder surgery include IV PCA, intraarticular injection, suprascapular nerve block (SSNB), single-shot ISB and CISB. An SSNB is superior to an intra-articular injection after shoulder arthroscopy but is inferior to ISB [6]. ISB is superior to a subacromial bursa block [7]. In particular, CISB consistently provides superior analgesia with fewer side effects than either continuous subacromial infusion [8] or single-shot
ISB. In addition, it allows earlier hospital discharge and possibly improves rehabilitation after major surgery $[9,10]$.

However, this technique requires the administration of large volumes of local anesthesia with the potential risk of toxicity due to the accumulation of the drug after prolonged periods of infusion [11]. Singlelyn et al. [12] reported that reducing the background infusion of $0.125 \%$ bupivacaine by half $(5 \mathrm{ml} / \mathrm{h}$ ) is associated with the use of small sized ( $2.5 \mathrm{ml} / 30 \mathrm{~min})$ PCA boluses, which provide excellent pain relief, no side effects, and a $36 \%$ decrease in local anesthetic consumption. For this reason, a technique consisting of a background infusion and PCA boluses was chosen.

There is little information regarding the optimal combination of the local anesthetic volume and concentration for CIBS when used for postoperative analgesia after shoulder surgery. In the absence of extensive data, the infusion settings for long-acting local anesthetics delivered to the brachial plexus include a basal rate of 5 to $10 \mathrm{ml} / \mathrm{h}$, a bolus volume of 2 to $5 \mathrm{ml} / \mathrm{h}$, and lockout duration of 20 to $60 \mathrm{~min}$. Some studies have supported the administration of relatively high volumes $(>5 \mathrm{ml} / \mathrm{h}$ ) of dilute local anesthetics, consistent with the large surface area of the brachial plexus in this region [5].

In this study, there was no difference between the groups with regard to the pain intensity. The pain scores at rest and on shoulder abduction were similar in the two groups in the recovery room, 24 and $48 \mathrm{~h}$ postoperatively. In addition, there were no differences in the proportion of patients requiring rescue analgesia and sleep disturbances due to pain between the groups. These results support the comparable pain relief between the groups.

One reason for the lack of differences in pain scores may be that the basal infusion volumes are too enough and may even work in catheters that are not located precisely at the nerves. To better determine the difference between a "well placed" and a "poorly placed" catheter, one should use smaller amounts of local anesthetics. Paqueron et al. [13] demonstrated that $2.7 \mathrm{ml}$ of mepivacaine $1.5 \%$ is sufficient to block the sciatic nerve in $50 \%$ of patients if the catheter tip is located precisely.

Another possible explanation for this finding is that the catheters were not placed as intimately to the C5-6 roots/ superior trunk in both groups. Therefore, the basal infusion volumes were too small. Hence, larger volumes will be needed for adequate pain control in both groups.

The relative importance of the local anesthetic concentration/ volume versus dose has significant clinical consequences given the wide range of local anesthetic concentrations used for perineural infusion $[5,14,15]$. Le et al. [16] reported that for CISB, a lower concentration of local anesthetic at a higher basal rate provided superior analgesia. Fredrickson et al. [17] reported that after major shoulder surgery, ropivacaine $0.2 \%$ at $2 \mathrm{ml} / \mathrm{h}$ 
with on-demand $5 \mathrm{ml}$ boluses administered via an ultrasoundguided C5-6 root/superior trunk perineural catheter produced similar analgesia, but greater patient satisfaction compared to those administered ropivacaine $0.4 \%$. On the other hand, Ilfeld et al. [18] reported that for continuous posterior lumbar plexus blocks, the local anesthetic concentration and volume do not affect the nerve block characteristics, suggesting that the local anesthetic dose (mass) is the primary determinant of perineural infusion effects. Although it is unlikely that there is a single optimal local anesthetic dose for all patients, there may be an optimal protocol for administering perineural local anesthetics (e.g., initial basal rate, bolus dose volume, lock-out duration, and subsequent adjustments). Future research should examine not only the optimal starting dose for various perineural catheter infusions but also the subsequent changes in dosing during the acute postoperative period.

A better sensorimotor dissociation of ropivacaine would be well suited to orthopedics because it may facilitate rehabilitation and improve the patient's well being. A smaller basal infusion was expected to be less likely to cause a motor block and facilitate rehabilitation. However, both groups showed similar degree of motor block.

Single-shot ISB is associated with ipsilateral hemidiaphragmatic paresis in all patients [19]. Patients undergoing CISB are also at risk of diaphragmatic paresis. During CISB, paresis can occur in as many as $75 \%$ of cases and often persists until the end of the infusion [20,21]. Yang et al. [22] reported a patient who developed atelectasis of the lung and pleural effusion manifesting as chest discomfort during CISB. The diaphragmatic function was not assessed specifically in this study, but 6 patients experienced mild dyspnea that was resolved after applying oxygen by a mask. However, 1 patient complained of moderate chest discomfort and dyspnea, which was resolved after discontinuing the infusion, and was excluded from the study.

In this study, two patients in group R8 reported new neurological symptoms after CISB, which were quite minor and resolved within 4 weeks. Perioperative nerve injury after upper extremity surgery may be the result of several contributing factors, either unrelated or related directly to the regional anesthetic technique. The unrelated risk factors include the patient and surgical issues. Regional anesthetic factors that may contribute directly to anesthesia-related nerve injury include mechanical trauma, ischemic injury, or chemical injury. Continuous catheter techniques raise concern regarding the potential neurotoxicity from repeated perineural injections of local anesthetics. One case of plexus irritation caused by an interscalene catheter was reported [23]. Borgeat et al. [24] reported a $11 \%$ incidence of neurological complications, mostly minor, 10 days after CISB, which decreased to $6,2.6$, and $0.4 \%$ after 1, 3 and 6 months, respectively. They also reported that CISB does not increase the risk of nerve injury compared to single-shot techniques.

The incidence of nausea and vomiting was infrequent and similar in both groups, and within the range of previous results [2]. The degree of patient satisfaction was high in the two groups and comparable to that reported in previous trials $[1,2]$. The high satisfaction of patients with the pain therapy in both groups shows the high quality of the CISB for additional regional anesthesia in shoulder surgery.

In this study, there were no medical complications attributable to the initial regional block, catheter placement or perineural infusion. However, the small number of patients means definite conclusions regarding its relative safety cannot be drawn.

There were some limitations regarding the study protocol, which must be considered when interpreting this data. First, the subjects and investigators were not masked to the treatment group. The pump for CISB continuously displays the reservoir volume, and although not instructed on how to do this, some patients may have determined their basal rate using this information, which may have compromised the double-blinded nature of the study. However, it is unlikely that patients had a bias toward one basal infusion rate or the other.

Second, interscalene catheters were placed for a range of procedures, each expected to be associated with a different postoperative pain intensity, which may have limited the ability of the study to detect a difference in postoperative pain between the treatment groups.

Third, 3 patients underwent surgery with general anesthesia. It is not believed that these low patients given general anesthesia might have influenced the results because they received similar doses of sufentanil and more doses of propofol to maintain anesthesia, the effects of which would rapidly disappear.

Fourth, the number of patient-controlled bolus doses administered was unavailable. The portable infusion pump described in this study cannot record the infusion/bolus details. This weakness might decrease the level of confidence in these results. Future studies will correct for the lack of total local anesthetic required in each treatment group.

Fifth, only pain intensity at the PACU, and 24 and $48 \mathrm{~h}$ after surgery was assessed. Breakthrough pain after resolution of the primary block generally occurs during 24 hours after surgery. Therefore, a more accurate comparison will require a shorter assessment interval.

In conclusion, after shoulder surgery, CISB with $0.2 \%$ ropiva-

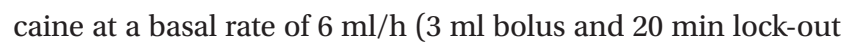
interval) produced similar analgesia, supplemental analgesia, motor block, adverse events and patient's satisfaction to that produced at a basal rate of $8 \mathrm{ml} / \mathrm{h}$. Therefore, decreasing the 
basal rate of CISB is more appropriate considering the side effects of local anesthetics. However, further studies will be needed to determine the optimal basal infusion volumes of ropivacaine in CISB.

\section{References}

1. Borgeat A, Schappi B, Biasca N, Gerber C. Patient-controlled analgesia after major shoulder surgery: patient-controlled interscalene analgesia versus patient-controlled analgesia. Anesthesiology 1997; 87: 1343-7.

2. Borgeat A, Tewes E, Biasca N, Gerber C. Patient-controlled interscalene analgesia with ropivacaine after major shoulder surgery: PCIA vs PCA. Br J Anaesth 1998; 81: 603-5.

3. Knudsen K, Beckman Suurkula M, Blomberg S, Sjovall J, Edvardsson N. Central nervous and cardiovascular effects of i.v. infusions of ropivacaine, bupivacaine and placebo in volunteers. Br J Anaesth 1997; 78: 507-14.

4. Borgeat A, Kalberer F, Jacob H, Ruetsch YA, Gerber C. Patientcontrolled interscalene analgesia with ropivacaine $0.2 \%$ versus bupivacaine $0.15 \%$ after major open shoulder surgery: the effects on hand motor function. Anesth Analg 2001; 92: 218-23.

5. Ilfeld BM, Morey TE, Wright TW, Chidgey LK, Enneking FK. Interscalene perineural ropivacaine infusion:a comparison of two dosing regimens for postoperative analgesia. Reg Anesth Pain Med 2004; 29: 9-16.

6. Singelyn FJ, Lhotel L, Fabre B. Pain relief after arthroscopic shoulder surgery: a comparison of intraarticular analgesia, suprascapular nerve block, and interscalene brachial plexus block. Anesth Analg 2004; 99: 589-92.

7. Laurila PA, Lopponen A, Kanga-Saarela T, Flinkkila T, Salomaki TE. Interscalene brachial plexus block is superior to subacromial bursa block after arthroscopic shoulder surgery. Acta Anaesthesiol Scand 2002; 46: 1031-6.

8. Delaunay L, Souron V, Lafosse L, Marret E, Toussaint B. Analgesia after arthroscopic rotator cuff repair: subacromial versus interscalene continuous infusion of ropivacaine. Reg Anesth Pain Med 2005; 30: 117-22.

9. Ilfeld BM, Vandenborne K, Duncan PW, Sessler DI, Enneking FK, Shuster JJ, et al. Ambulatory continuous interscalene nerve blocks decrease the time to discharge readiness after total shoulder arthroplasty: a randomized, triple-masked, placebo-controlled study. Anesthesiology 2006; 105: 999-1007.

10. Ilfeld BM, Wright TW, Enneking FK, Morey TE. Joint range of motion after total shoulder arthroplasty with and without a continuous interscalene nerve block: a retrospective, case-control study. Reg Anesth Pain Med 2005; 30: 429-33.

11. Tuominen M, Pitkanen M, Rosenberg PH. Postoperative pain relief and bupivacaine plasma levels during continuous interscalene brachial plexus block. Acta Anaesthesiol Scand 1987; 31: 276-8.

12. Singelyn FJ, Seguy S, Gouverneur JM. Interscalene brachial plexus analgesia after open shoulder surgery: continuous versus patientcontrolled infusion. Anesth Analg 1999; 89: 1216-20.

13. Paqueron X, Narchi P, Mazoit JX, Singelyn F, Benichou A, Macaire P. A randomized, observer-blinded determination of the median effective volume of local anesthetic required to anesthetize the sciatic nerve in the popliteal fossa for stimulating and nonstimulating perineural catheters. Reg Anesth Pain Med 2009; 34: $290-5$.

14. Sandefo I, Bernard JM, Elstraete V, Lebrun T, Polin B, Alla F, et al. Patient-controlled interscalene analgesia after shoulder surgery: catheter insertion by the posterior approach. Anesth Analg 2005; 100: 1496-8.

15. Borgeat A, Blumenthal S, Lambert M, Theodorou P, Vienne P. The feasibility and complications of the continuous popliteal nerve block: a 1001-case survey. Anesth Analg 2006; 103: 229-33.

16. Le LT, Loland VJ, Mariano ER, Gerancher JC, Wadhwa AN, Renehan $\mathrm{EM}$, et al. Effects of local anesthetic concentration and dose on continuous interscalene nerve blocks: a dual-center, randomized, observer-masked, controlled study. Reg Anesth Pain Med 2008; 33: 518-25.

17. Fredrickson MJ, Price DJ. Analgesic effectiveness of ropivacaine $0.2 \%$ vs $0.4 \%$ via an ultrasound-guided C5-6 root/superior trunk perineural ambulatory catheter. Br J Anaesth 2009; 103: 434-9.

18. Ilfeld BM, Moeller LK, Mariano ER, Loland VJ, Stevens-Lapsley JE, Fleisher AS, et al. Continuous peripheral nerve blocks: is local anesthetic dose the only factor, or do concentration and volume influence infusion effects as well? Anesthesiology 2010; 112: 347-54.

19. Urmey WF, Talts KH, Sharrock NE. One hundred percent incidence of hemidiaphragmatic paresis associated with interscalene brachial plexus anesthesia as diagnosed by ultrasonography. Anesth Analg 1991; 72: 498-503.

20. Tuominen M, Tarkkila P. Diaphragmatic motion during continuous interscalene brachial plexus block. Reg Anesth 1995; 20: 543-4.

21. Pere P, Pitkanen M, Rosenberg PH, Bjorkenheim JM, Linden H, Salorinne Y, et al. Effect of continuous interscalene brachial plexus block on diaphragm motion and on ventilatory function. Acta Anaesthesiol Scand 1992; 36: 53-7.

22. Yang CW, Jung SM, Cho CK, Kwon HU, Kang PS, Lim YS, et al. Pleural effusion and atelectasis during continuous interscalene brachial plexus block -A case report. Korean J Anesthesiol 2010; 58: 95-8.

23. Ribeiro FC, Georgousis H, Bertram R, Scheiber G. Plexus irritation caused by interscalene brachial plexus catheter for shoulder surgery. Anesth Analg 1996; 82: 870-2.

24. Borgeat A, Ekatodramis G, Kalberer F, Benz C. Acute and nonacute complications associated with interscalene block and shoulder surgery: a prospective study. Anesthesiology 2001; 95: 875-80. 